\title{
Efficacy of confronting smokers with airflow limitation for smoking cessation
}

\author{
D. Kotz*, G. Wesseling ${ }^{\#}$, M.J.H. Huibers ${ }^{\star}$ and O.C.P. van Schayck*
}

ABSTRACT: The objective of the present study was to test whether confronting smokers with previously undetected chronic obstructive pulmonary disease (COPD) increases the rate of smoking cessation.

In total, 296 smokers with no prior diagnosis of COPD were detected with mild-to-moderate airflow limitation by means of spirometry and randomly allocated to: confrontational counselling by a nurse with nortriptyline for smoking cessation (experimental group); regular counselling by a nurse with nortriptyline (control group 1); or "care as usual" for smoking cessation by the general practitioner (control group 2). Only the experimental group was confronted with their abnormal spirometry (mean forced expiratory volume in one second (FEV1) post-bronchodilator $80.5 \%$ predicted, mean FEV1/forced vital capacity post-bronchodilator $62.5 \%$ ).

There was no difference in cotinine-validated prolonged abstinence rate between the experimental group (11.2\%) and control group 1 (11.6\%) from week 5-52 (odds ratio (OR) 0.96, $95 \%$ confidence interval $(\mathrm{Cl}) \mathbf{0 . 4 3 - 2 . 1 8}$ ). The abstinence rate was approximately twice as high in the experimental group compared with control group $2(5.9 \%)$, but this difference was not statistically significant (OR $2.02,95 \% \mathrm{CI} 0.63-6.46)$.

The present study did not provide evidence that the confrontational approach increases the rate of long-term abstinence from smoking compared with an equally intensive treatment in which smokers were not confronted with spirometry. The high failure rates $(\geqslant 88 \%)$ highlight the need for treating tobacco addiction as a chronic relapsing disorder.

KEYWORDS: Chronic obstructive pulmonary disease, confrontational counselling, respiratory nurse, smoking cessation, spirometry

hronic obstructive pulmonary disease (COPD) is a preventable and treatable disease which is characterised by airflow limitation that is not fully reversible [1]. Spirometry is the gold standard for the diagnosis and assessment of the disease [1]. COPD is currently the fifth leading cause of death worldwide [2], and projections for 2020 indicate a further increase in global mortality, placing COPD in the third position of lethal diseases [3]. Cigarette smoking is by far the most important risk factor for COPD, and smoking cessation is the single most effective way to reduce the risk of developing COPD and to affect the outcome in patients at all stages of the disease $[4,5]$.

Under diagnosis of COPD is a worldwide problem [6]. Most patients present to their doctor for various other reasons but often have respiratory symptoms, and in those who do present with respiratory symptoms, COPD is not always suspected or diagnosed [7]. Because of the irreversible and progressive nature of the disease, early intervention is important. However, the use of spirometry for early detection of airflow limitation and COPD is still being debated [810]. The most important counter-argument is that there is no convincing evidence that spirometry increases smoking cessation rates [11-13].

Discussing abnormal test results with smokers has been suggested to be a "teachable moment" that may increase motivation to quit smoking, but there is only weak evidence to support such an approach [14]. Various studies have been performed on the efficacy of spirometry as a motivational tool for smoking cessation but the results are inconclusive $[11,12]$. Findings are often of limited validity because of one or more important biases, such as unstandardised counselling intensity, incomparable or uncontrolled use of pharmacological aids for smoking cessation between the experimental and control group, or different (or unclear) baseline levels of lung function and motivation to quit smoking [15]. The most recent randomised trial clearly showed a positive effect; telling smokers
AFFILIATIONS

*Dept of General Practice, School for Public Health and Primary Care (CAPHRI), Maastricht University Medical Centre

\#Dept of Respiratory Medicine, Maastricht University Medical Centre, and

'Dept of Clinical Psychological Science, Maastricht University, Maastricht, The Netherlands.

\section{CORRESPONDENCE}

D. Kotz, Dept of General Practice, School for Public Health and Care (CAPHRI), Maastricht University Medical Centre, P.0. Box 616, 6200 MD Maastricht,

The Netherlands

Fax: 31433619344

E-mail: d.kotz@hag.unimaas.nl

Received:

November 302008

Accepted after revision:

December 052008

SUPPORT STATEMENT

This study was funded by grants from the Dutch Asthma Foundation,

Partners in Care Solutions for COPD (an initiative of the School for Primary Care and Public Health (CAPHRI) Boehringer Ingelheim and Pfizer), and Maastricht University Medical Centre. The funders were not involved in the study design; in the collection, analysis, and interpretation of data; in the writing of the report; or in the decision to submit the article for publication. This study is registered at the Netherlands Trial Register (ISRCTN 64481813).

STATEMENT OF INTEREST Statements of interest for $G$. Wesseling and O.C.P. van Schayck and the study itself can be found at www. erj.ersjournals.com/misc/ statements.dtl 
their "lung age" (based on spirometry) increased the abstinence rate by $7.2 \%$ after 52 weeks [16].

The present authors hypothesised that early detection of COPD and confrontation with spirometry for smoking cessation may be effective if "confrontational counselling" is applied [17] Confrontational counselling is a patient-centred approach that involves confronting smokers with the consequences of their addiction (previously undiagnosed COPD) and which uses specific communication skills to identify and challenge irrational beliefs about smoking. A randomised controlled trial was conducted in current smokers with previously undiagnosed mild-to-moderate airflow limitation to assess the efficacy of confrontational counselling in comparison with regular health education and promotion for smoking cessation delivered by specialised respiratory nurses, with regard to prolonged abstinence from smoking rates from week 5-52 after the target cessation date. Secondary outcomes were abstinence rates at week 5 and from week 5-26.

\section{METHODS}

The trial was designed to assess the "net" effect of confronting smokers with spirometry by comparing medium-intensity confrontational counselling delivered by a respiratory nurse combined with nortriptyline for smoking cessation (experimental group) with medium-intensity health education and promotion delivered by a respiratory nurse combined with nortriptyline for smoking cessation (control group 1). The effect of both treatments were compared to low-intensity "care as usual" for smoking cessation by the general practitioner (GP; control group 2). A detailed description of the protocol has been published previously [18]. The trial was approved by the medical ethics committee of Maastricht University Medical Centre (Maastricht, the Netherlands) and registered at the Netherlands Trial Register (ISRCTN 64481813).

\section{Recruitment and eligibility of participants}

Current smokers aged 35-70 yrs who were interested in quitting were recruited from the general population (through advertisements in local newspapers, flyers, posters and mailings to households) and from primary care practices (during consultations and through posters in the waiting room and personalised mailings) in Dutch- and Belgian-Limburg (the region surrounding Maastricht). The text from the advertisements, flyers and posters explained that Maastricht University performs a study on smoking cessation treatment in which individual behavioural support is combined with medication for smoking cessation. No information was given to participants during recruitment about the desired target condition (airflow limitation).

Eligibility was assessed during an initial telephone interview. Inclusion criteria were: smoking history of $\geqslant 10$ pack-yrs (number of cigarettes smoked per day multiplied by number of years smoking then divided by 20); being competent in reading and speaking Dutch; and reporting at least one of the respiratory symptoms (cough, sputum production or shortness of breath). Exclusion criteria were: evidence of a prior respiratory diagnosis, defined by an affirmative answer to the question "Do you have COPD, chronic bronchitis, asthma or asthmatic bronchitis?". Subjects were also excluded if they had undergone spirometry during the preceding 12 months.
One or more contraindications for using the smoking cessation medication (nortriptyline) were also criteria for exclusion; among others was the current use of antidepressants.

After the initial telephone interview, the participant information sheet with the informed consent form and the baseline questionnaire were sent to eligible subjects, and a date was arranged for spirometry testing at the Medical Centre Annadal (Maastricht). The participant information sheet did not include any information about early detection and confrontation with COPD or the differences in counselling between the experimental and control group 1. The design used was adapted from the design by ZELEN $[19,20]$, which may be particularly useful when evaluating the full unbiased impact of screening interventions [21].

Spirometry was performed according to American Thoracic Society/European Respiratory Society criteria [22, 23] using a Vitalograph $_{\circledast} 2120$ (Vitalograph Ltd, Buckingham, UK). Final eligibility was determined if subjects had airflow limitation defined as post-bronchodilator forced expiratory volume in one second (FEV1) / forced vital capacity (FVC) $<70 \%$ in combination with post-bronchodilator $\mathrm{FEV} 1 \geqslant 50 \%$ predicted, i.e. mild or moderate airflow limitation, according to the international Global Initiative for Chronic Obstructive Lung Disease (GOLD) guidelines [1]. The results of spirometry were not discussed at that time. Subjects with severe airflow limitation (post-bronchodilator FEV1 $<50 \%$ pred) were excluded from participation and advised to contact their GP or a lung physician for further evaluation. Subjects without airflow limitation (post-bronchodilator $\mathrm{FEV} 1 / \mathrm{FVC}>70 \%$ ) were also excluded. All excluded smokers were told that despite their normal lung function, they still were at risk of getting other smoking-related diseases that are not measured by spirometry, such as cancer or cardiovascular disease. They were strongly advised to give up smoking. After the last follow-up measurement, all participants received a debriefing letter with detailed information about the study and their GPs were informed about the results from spirometry.

All eligible subjects were contacted by telephone a few days after baseline spirometry to be randomised into one of the three intervention groups. The database of the trial incorporated a randomisation system of seven participants per block, allowing an unequal group allocation of 3:3:1 for experimental group:control group 1:control group 2 (the trial initially began with an equal group allocation of $1: 1: 1$ but this was then changed to $3: 3: 1$ ).

\section{Interventions}

Participants from both the experimental group and control group 1 received medium-intensity counselling delivered by a respiratory nurse and combined with nortriptyline for smoking cessation. The common basis for the counselling in both groups was the so-called "L-MIS" protocol for the treatment of nicotine and tobacco addiction which has been implemented among all respiratory nurses in the Netherlands in previous years [24]. The number of counselling sessions $(n=4)$, their duration (40 $\mathrm{min}$ ) and scheduling (weeks 1, 2, 3 and 4) was standardised in both treatment groups (table 1). Participants' attendance of counselling was assessed by counting the number of counselling sessions attended and dividing this number by four. Specific elements of confrontational counselling [17] were added to the L-MIS in the experimental group, 
which discriminated the treatment from that in control group 1 (table 1). This involved discussing the results from spirometry and the prognosis of COPD, and challenging irrational beliefs about smoking.

Participants from the experimental group and control group 1 received an equal dosage of nortriptyline for smoking cessation. Due to this, the pharmacological treatment component was standardised and the risk of co-interventions used by participants was reduced. Nortriptyline is a tricyclic antidepressant and was chosen because it has been shown to be a cheap and effective alternative for the antidepressant bupropion [26, 27]. Participants started taking nortriptyline on the day of the first counselling session (day 1). From day 1-3,

\section{TABLE 1 Components of counselling in experimental group and control group 1}

General components in both experimental group and control group

FC1 day 1: 40 min counselling by RN

Assess and discuss smoking characteristics

Assess and increase motivation to quit

Discuss cons of smoking and pros of cessation

Start use of nortriptyline

FC2 day 8: 40 min counselling by RN

Evaluate use of nortriptyline

Assess and increase self-efficacy to quit

Prepare the TQD

Anticipate barriers to cessation and withdrawal

TQD: TC day 14: 5 min counselling by RN

Evaluate quit attempt

Give advice about cessation and abstaining

FC3 day 15: 40 min counselling by $\mathrm{RN}$

Evaluate cessation attempt

Evaluate use of nortriptyline

Give advice about relapse prevention

FC4 day 22: 40 min counselling by RN

Evaluate cessation attempt

Evaluate use of nortriptyline

Give advice about relapse prevention

End counselling

Additional components of confrontational counselling in the experimental group only

Incorporated in $\mathrm{FC} 1+2$

Discuss the spirometry results

Confront the consequences of smoking: the diagnosis COPD

Discuss the severity and prognosis of COPD and the benefits of smoking cessation by using the "Fletcher curve" and images of normal and smoker's lungs [25]

Incorporated in FC3+4

Reflect on the smoker's thoughts, feelings and beliefs about COPD

Challenge irrational beliefs about smoking by raising the smoker's consciousness about these beliefs, testing their reality and by exploring the relationship between beliefs and behaviour

Use a smoking cessation diary to monitor smoking behaviour and beliefs about smoking

FC: face-to-face counselling session; RN: respiratory nurse; TQD: target quit date; TC: telephone counselling session; COPD: chronic obstructive pulmonary disease. participants took a 25-mg nortriptyline tablet once a day (preferably after dinner). From day 4-7, participants took $50 \mathrm{mg}$ a day (administered as two 25-mg tablets). From day 8 to the end of the treatment period (day 49), participants took $75 \mathrm{mg}$ a day (administered as three 25-mg tablets). The nurse monitored the correct use of the medication and the occurrence of side-effects. In case of unpleasant or severe side-effects, the dosage was reduced or the use of the medication was stopped.

To test whether the experimental intervention as a whole was more effective than primary care as usual, participants from control group 2 were referred to their own GP for smoking cessation treatment. They were asked to make an appointment with their GP within the next 10 days. They were provided with a referral letter explaining to the GP that they were participating in a study on smoking cessation. This letter did not give any information about the spirometry results or the fact that the participant had airflow limitation. The GP was asked to provide the care usually provided to patients who want to quit smoking. In the Netherlands, standard primary care for smoking cessation involves the use of a protocol for low-intensity health education and promotion, the so-called "H-MIS" [28]. A semistructured interview was used among participants from control group 2 during the first follow-up visit in order to assess whether participants had indeed consulted their GP and which treatment the GP had delivered for smoking cessation.

\section{Outcome measures and sample size}

The primary outcome measure was prolonged abstinence from smoking from week 5-52 after the target quit date. Prolonged abstinence was defined as urine cotinine-validated $\left(<50 \mathrm{ng} \cdot \mathrm{mL}^{-1}\right.$ [29]) abstinence from smoking at all three follow-up visits at 5, 26 and 52 weeks. The calculation of the sample size was based on the identification of a difference in prolonged abstinence rates of 15\%: $35 \%$ quitters in the experimental group versus $20 \%$ in control group 1 . This resulted in 136 participants needed in both groups $(\alpha=0.05, \beta=0.20)$ [30]. A larger difference was expected between the experimental group (35\%) and control group 2 (8\%) [28] and, therefore, fewer participants were needed in control group $2(n=32)$.

Participants completed a questionnaire at baseline and at each follow-up visit. The questionnaire included various smoking characteristics and the Fagerström Test for Nicotine Dependence (FTND) [31]. Respiratory health complaints were measured with the Clinical COPD Questionnaire [32, 33]. Health-related quality of life was measured with the selfreported Chronic Respiratory Questionnaire [34, 35].

\section{Data analyses}

Statistical differences in abstinence from smoking rates were analysed using simple logistic regression analyses to calculate odds ratios (ORs) and 95\% confidence intervals (CIs). As part of an ancillary analysis, multiple logistic regression models were used to adjust for baseline covariates that are known to be associated with the primary outcome [36]: age, sex, level of education, number of previous cessation attempts, anxiety (measured with the hospital anxiety and depression scale [37]), and nicotine addiction (measured with the FTND). Furthermore, a subgroup analysis was performed to compare the abstinence rates of smokers with mild versus moderate COPD. All randomised subjects were included in the intention-to-treat 


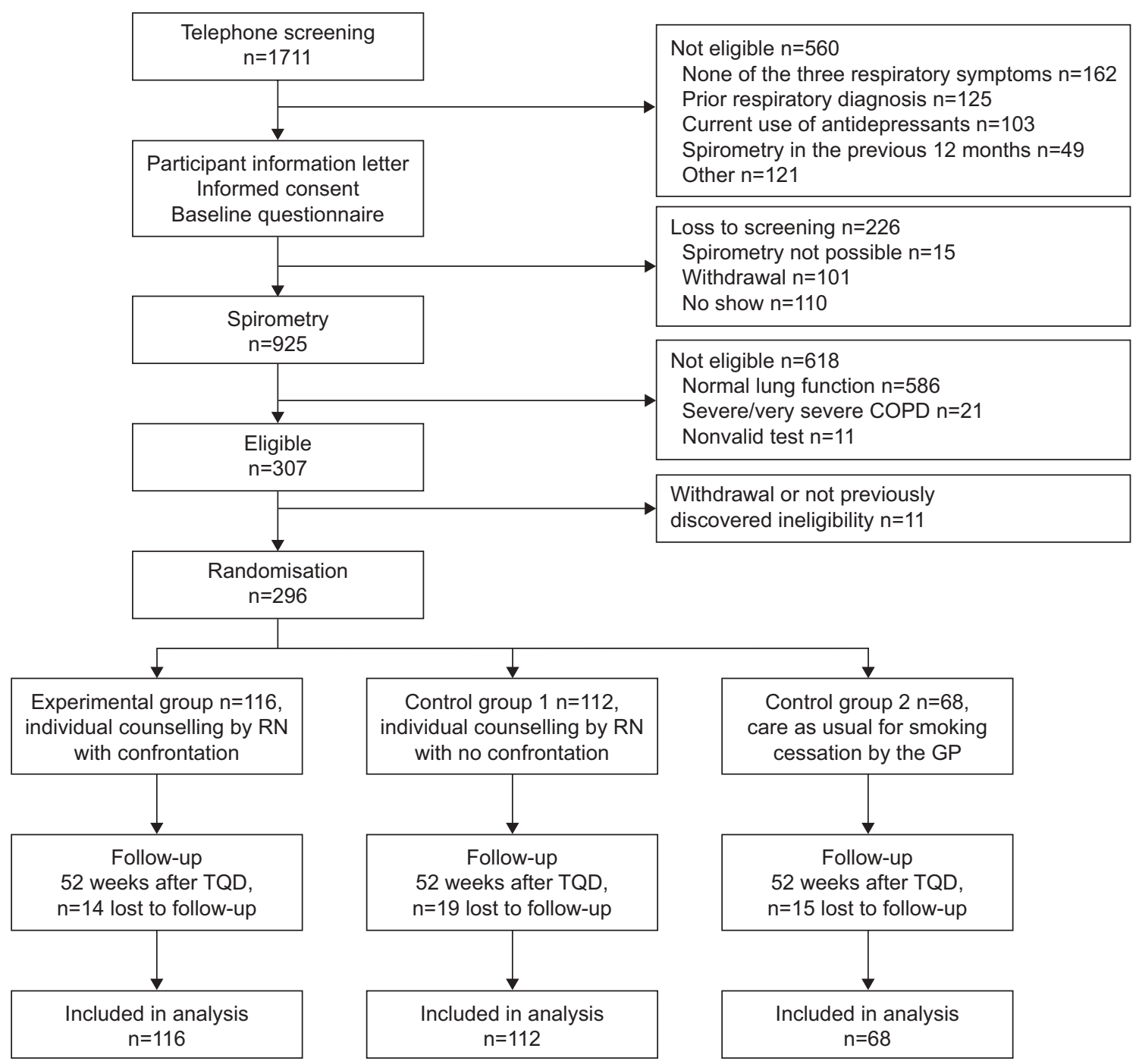

FIGURE 1. Study flowchart. COPD: chronic obstructive pulmonary disease; RN: respiratory nurse; GP: general practitioner; TQD: target quit date.

analyses, and subjects not attending the follow-up visit or with a missing value on the measure of abstinence were regarded as smokers. The proportion of missing data on items from the questionnaire ranged $0-7 \%$. Missing data were not imputed for any analyses.

\section{RESULTS}

A total of 116 smokers with previously undetected COPD were randomly allocated to the experimental group, 112 to control group 1 and 68 to control group 2 (fig. 1). After 52 weeks follow-up, the numbers of participants lost to follow-up (i.e. with no data on the primary outcome variable) were: $14(12 \%)$, $19(17 \%)$ and $15(22 \%)$ in the experimental group, control group 1 and control group 2, respectively. The baseline characteristics are shown in table 2 . A total of $160(54 \%)$ participants had mild COPD and 136 (46\%) had moderate COPD according to the GOLD classification.

\section{Treatment received}

One participant from the experimental group and one participant from control group 1 dropped out before the start of the counselling because they already had stopped smoking. Among the remaining participants, attendance in the counselling sessions was $95 \%$ in the experimental group and $92 \%$ in control group 1 (no statistically significant difference). The proportion of participants reporting one or more side-effect of using nortriptyline to the respiratory nurse was 82,84 and $72 \%$ during counselling sessions 2, 3 and 4, respectively, (no statistically significant difference between the groups). The mean number of side-effects reported was lower in the experimental group (mean 1.4) than in control group 1 (mean $1.8 ; \mathrm{p}=0.017)$. Among all participants reporting side-effects, dry mouth was most frequently reported $(39 \%)$, followed by fatigue $(10 \%)$ and dizziness $(10 \%)$

Of the 68 participants from control group 2 who were referred to their GP for care as usual for smoking cessation, $46(68 \%)$ actually consulted their GP, four $(6 \%)$ did not and no information was available for 18 (27\%) participants. Among the 46 participants who consulted their GP, the median number of consultations was two (maximum five). The median (range) duration of these consultations was 10 (5-45) min. 


\begin{tabular}{|c|c|c|c|}
\hline Characteristic & Experimental group & Control group 1 & Control group 2 \\
\hline Age yrs & $53.8 \pm 7.0$ & $54.9 \pm 8.0$ & $53.0 \pm 7.6$ \\
\hline Males & $71(61.2)$ & $74(66.1)$ & $40(58.8)$ \\
\hline Level of educational background ${ }^{\#}$ & $3.9 \pm 1.7$ & $3.8 \pm 1.8$ & $4.0 \pm 1.9$ \\
\hline Cigarettes per day & $23.9 \pm 8.4$ & $23.2 \pm 9.9$ & $22.7 \pm 9.6$ \\
\hline Smoking history pack-yrs & $44.1 \pm 18.3$ & $44.2 \pm 19.1$ & $41.5 \pm 19.7$ \\
\hline Nicotine dependence FTND ${ }^{+}$ & $4.6 \pm 1.5$ & $4.5 \pm 1.5$ & $4.4 \pm 1.5$ \\
\hline Number of previous cessation attempts & $3.5 \pm 3.5$ & $4.0 \pm 5.7$ & $4.2 \pm 3.3$ \\
\hline \multicolumn{4}{|l|}{ Previous use of pharmacotherapy for smoking cessation } \\
\hline Nicotine gum & $30(25.9)$ & $36(32.1)$ & $30(44.1)$ \\
\hline \multicolumn{4}{|l|}{ Disease specific quality of life $\mathbf{C R Q}-\mathbf{S R}^{f}$} \\
\hline Fatigue & $4.7 \pm 1.1$ & $4.7 \pm 1.3$ & $4.6 \pm 1.4$ \\
\hline Emotional function & $4.8 \pm 1.1$ & $4.8 \pm 1.2$ & $4.8 \pm 1.1$ \\
\hline Mastery & $5.0 \pm 0.8$ & $5.0 \pm 0.8$ & $5.0 \pm 0.8$ \\
\hline Anxiety HADS $\# \#$ & $5.6 \pm 3.7$ & $6.0 \pm 3.9$ & $5.9 \pm 4.1$ \\
\hline FEV1 post-bronchodilator \% pred & $80.5 \pm 14.7$ & $83.7 \pm 16.8$ & $79.7 \pm 14.0$ \\
\hline FVC post-bronchodilator \% pred & $103.9 \pm 14.9$ & $107.6 \pm 17.8$ & $105.4 \pm 14.4$ \\
\hline FEV1/FVC post-bronchodilator & $62.5 \pm 5.9$ & $63.0 \pm 6.1$ & $61.9 \pm 6.3$ \\
\hline \multicolumn{4}{|l|}{ GOLD classification } \\
\hline GOLD 1 & $62(53.4)$ & $66(58.9)$ & $32(47.1)$ \\
\hline GOLD 2 & $54(46.6)$ & $46(41.1)$ & $36(52.9)$ \\
\hline
\end{tabular}

Data are presented as mean \pm SD or $\mathrm{n}(\%)$, unless otherwise stated. BMI: body mass index; FTND: Fagerström Test for Nicotine Dependence; COPD: chronic obstructive pulmonary disease; CCQ: Clinical COPD Questionnaire; CRQ: Chronic Respiratory Questionnaire; SR: self rated; HADS: hospital anxiety and depression scale; FEV1: forced expiratory volume in one second; \% pred: \% predicted; FVC: forced vital capacity; GOLD: Global Initiative for Chronic Obstructive Lung Disease. \#: ranges from 1 (lowest education) to 7 (highest); $": 1$ pack-yr is the number of cigarettes smoked per day multiplied by number of years of smoking divided by $20 ;{ }^{+}:$ranges from 0 (lowest level of nicotine dependence) to 10 (highest); ${ }^{\text {s: }}$ ranges from 0 (very good control of COPD) to 6 (very poor); ${ }^{f}$ : ranges per subdomain from 1 (maximum impairment) to 7 (minimum); ${ }^{\# \#}$ : ranges from 0 (lowest degree of anxiety) to 21 (highest).

Antidepressants were prescribed for smoking cessation in 34 out of the 46 participants: bupropion, $n=15$; nortriptyline, $\mathrm{n}=17$; or amitriptyline, $\mathrm{n}=1$. The type of antidepressant was unknown in one participant. Nicotine replacement therapy was prescribed in six out of the 46 participants.

\section{Abstinence from smoking}

The abstinence from smoking rates in the experimental group, control group 1 and control group 2 dropped from $51 \%$
(59 out of 116), 39\% (44 out of 112) and 18\% (12 out of 68 ), respectively, at week 5 after the target quit date to $11 \%$ (13 out of 116), $12 \%$ (13 out of 112), and $6 \%$ (four out of 68 ), respectively, from week 5-52 (table 3, fig. 2). The odds of being abstinent from smoking was $60 \%$ higher in the experimental group than in control group 1 at week 5 (OR $1.60,95 \%$ CI $0.95-2.70$ ) and 43\% higher from week 5-26 (OR $1.43,95 \%$ CI $0.79-2.58)$. There was no difference in prolonged abstinence rates from week 5-52 (OR 0.96, 95\% CI 0.43-2.18;

\section{TABLE 3 Cotinine-validated abstinence from smoking rates}

\begin{tabular}{lcccccc} 
Weeks from TQD $\mathbf{n}$ & Experimental group & $\mathbf{C 1}$ & $\mathbf{C 2}$ & Experimental versus $\mathbf{C 1}$ & Experimental versus C2 & C1 versus C2 \\
\hline $\mathbf{5}$ & $59(50.9)$ & $44(39.3)$ & $12(17.6)$ & $1.60(0.95-2.70), p=0.080$ & $4.83(2.35-9.94), p<0.001$ & $3.02(1.46-6.26), p=0.003$ \\
$\mathbf{5 - 2 6}$ & $35(30.2)$ & $26(23.2)$ & $8(11.8)$ & $1.43(0.79-2.58), p=0.236$ & $3.24(1.40-7.49), p=0.006$ & $2.27(0.96-5.35), p=0.062$ \\
$\mathbf{5 - 5 2}$ & $13(11.2)$ & $13(11.6)$ & $4(5.9)$ & $0.96(0.43-2.18), p=0.961$ & $2.02(0.63-6.46), p=0.236$ & $2.10(0.66-6.73), p=0.211$ \\
\hline
\end{tabular}

Data are presented as $n(\%)$ or odds ratio for abstinence (95\% confidence interval), p-value. The odds ratios are unadjusted for baseline covariates. TQD: target quit date; C1: control group 1; C2: control group 2. 


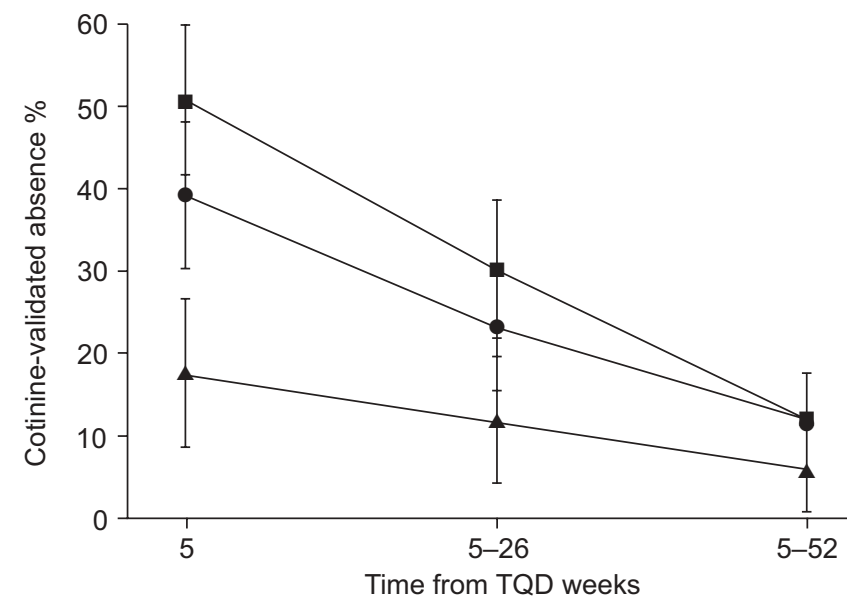

FIGURE 2. Cotinine-validated abstinence from smoking rates up to 52 weeks after the target quit date (TQD). $\mathbf{\square}$ : experimental group; $\bullet$ : control group 1; $\boldsymbol{\Delta}$ control group 2.

table 3). The corresponding ORs adjusted for baseline covariates were as follows: week 5 (OR 2.01, 95\% CI 1.1-3.7); week 5-26 (OR $1.58,95 \%$ CI 0.82-3.03); and week 5-52 (OR 0.88, 95\% CI 0.38 2.03). Compared to control group 2 , the odds of being abstinent from smoking from week 5-52 was approximately twice as high in the experimental group and control group 1, but these differences were not statistically significant (table 3). However, both at week 5 (OR 4.83) and from week 5-26 (OR 3.24), the odds of abstinence from smoking was significantly higher in the experimental group compared with control group 2 .

Across the total study group, differences in abstinence rates between smokers with mild COPD versus smokers with moderate COPD were small and not statistically significant. At week 5 after the target quit date, $40 \%$ (64 out of 160) of smokers with mild COPD were abstinent from smoking compared with $38 \%$ (51 out of 136) of smokers with moderate COPD. The corresponding abstinence rates were $25 \%$ (40 out of 160) versus $21 \%$ ( 29 out of 136 ) from week $5-26$, and $11 \%$ (17 out of 160 ) versus $10 \%$ (13 out of 136 ) from week $5-52$. There were also no significant differences within each of the three treatment groups.

\section{DISCUSSION}

A randomised controlled trial was conducted in 296 smokers with previously undetected mild-to-moderate COPD to assess the efficacy of confronting smokers with the results of spirometry for smoking cessation. Although the present authors observed a clinically relevant but statistically nonsignificant difference at week 5 , the confrontational counselling approach did not increase the prolonged abstinence from smoking rate from week 5-52 compared with an equally intensive treatment in which participants were not confronted with spirometry. In both groups, the proportion of smokers that did not succeed to quit or relapsed was very high $(\sim 88 \%)$.

The use of spirometry for early detection of COPD is an issue of debate, primarily because of a lack of convincing evidence showing that spirometry has an added positive effect on smoking cessation [11-13]. The results from previous studies $[8,11,12]$ are inconclusive, but the most recent study [16] shows a clear positive effect. PARKES et al. [16] evaluated the impact of telling smokers their estimated lung age after spirometry. Contrary to the current findings, significantly more smokers from the intervention group than from the control group were abstinent from smoking after 52 weeks: 13.6 versus $6.4 \%$. PARKES et al. [16] concluded that "telling smokers their lung age significantly improves the likelihood of them quitting smoking."

Why did PARKes et al. [16] find an effect whereas the present authors did not? This could be explained, in a large part, by the differences between the two studies in the recruitment strategy and resulting characteristics of the study samples. First, subjects from the study by PARKES et al. [16] had a better lung function (mean FEV1 90\% pred and mean FEV1/FVC 75\% compared with mean FEV1 $82 \%$ pred and mean FEV1/FVC $63 \%$ in the present study) and approximately one third of those with abnormal lung function were already known to have COPD; however in the present study, smokers with a previous diagnosis of COPD were excluded. Thus, the two study samples are not comparable concerning their baseline risk of COPD, a factor that is likely to affect the treatment outcome. Furthermore, the recruitment strategy of PARKES et al. [16] probably led to a selection of participants who were interested in their lung function and were, therefore, more susceptible to related health warnings. This is the same mechanism that may explain the results from a large observational study in smokers from Poland which showed that spirometry promoted cessation [38]. Also in the Polish study [38], selection bias may have occurred towards a group of smokers that was more interested in their lung health with the result that discussing spirometry may have a greater impact on smoking cessation [39]. In the present study, participants responded to announcements to receive a smoking cessation intervention (no attention was drawn to lung function testing). Therefore, subjects may have been less susceptible to the health warnings. Another important point is that the present study controlled the smoking cessation interventions in the experimental group and control group 1 to assess the "net" effect of confronting smokers with COPD, whereas PARKES et al. [16] did not standardise the smoking treatments smokers used following confrontation with spirometry. It may be that these smokers made more use of, and were more compliant with, evidencebased treatments for smoking cessation. All these differences in recruitment strategies are very important when interpreting results. Using spirometry in average (and mostly healthy) smokers who are interested in their lung function may trigger an attempt to quit smoking (and increase the likelihood of smoking cessation) but in smokers with COPD who are interesting in cessation, confrontation with the results from smoking does not seem to be effective. Another point to be mentioned when comparing the results of the current study with those of PARKES et al. [16] is the limitation of the latter study to use a point-prevalence estimate as primary outcome: the rate of nonsmokers at 52 weeks. The present authors used three follow-up measurements to calculate prolonged abstinence from smoking rates from week 5-52, as recommended by the Society for Research on Nicotine and Tobacco [40]. Furthermore, the average age of the participants from the current study was comparable with the participants from the study of PARKES et al. [16], but the present study participants had a heavier smoking history: 44 pack-yrs compared with 
31 pack-yrs in the study of PARKES et al. [16]. This indicates higher levels of nicotine and tobacco addiction, which is associated with a lower likelihood of smoking cessation.

Taking a closer look at the results from the present study, it appears as if the confrontational counselling approach did have a short-term effect on smoking cessation. The abstinence rate at 5 weeks after the target quit date was almost $12 \%$ higher in the experimental group compared with control group 1 (OR 1.60), which is a clinically relevant difference for a comparison of two equally intensive treatments. The p-value was only marginally significant $(p=0.08)$, but would probably have reached a level below 0.05 when the sample size (and therefore the power) was bigger. After adjusting for baseline covariates, the OR increased to 2.01 with a p-value of 0.023 . These findings suggest that confrontation counselling was likely to have an effect until shortly after the completion of the counselling treatment but that this effect diminished during the follow-up period in which the participants did not receive any more counselling.

To the best of the current authors' knowledge, only two other trials have studied the efficacy of antidepressants for smoking cessation in smokers with COPD. TASHKIN et al. [41] performed a randomised trial on the efficacy of bupropion for smoking cessation in 404 smokers with COPD. The abstinence rate in the bupropion group after 26 weeks follow-up was 16\% (compared with $9 \%$ in the placebo group). WAGENA et al. [42] performed a randomised trial on the efficacy of bupropion and nortriptyline for smoking cessation in 255 smokers of which $56 \%$ had COPD. The abstinence rates after 26 weeks of followup were $27 \%$ in the bupropion subgroup with COPD and $21 \%$ in the nortriptyline subgroup with COPD (compared with $8 \%$ in the placebo group). The subgroup of smokers with COPD who received nortriptyline had a lower abstinence rate than the subgroup of smokers with normal lung function (32\%), indicating a lower likelihood of quitting in smokers with airflow limitation. These results are comparable with the results from the present study and underline the high relapse rates in smokers with COPD who try to quit smoking.

A major strength of the present study is that all factors which are known to be associated with abstinence from smoking were standardised in both the experimental group and control group 1: type of counsellor (respiratory nurse); type of counselling (face-to-face and by telephone); number and duration of counselling sessions; and type (nortriptyline) and dosage of smoking cessation medication. The baseline risk for COPD of all participants was the same; they all had previously undetected mild-to-moderate airflow limitation. Only participants from the experimental group were confronted with their disease and, therefore, the "net" effect of confronting and counselling smokers with COPD could be assessed. A potential limitation of this approach is that the intensity and the standardisation of the counselling and the use of smoking cessation medication in the two groups may have diluted the specific effect of the information about lung damage. However, it may be that the cognitive change the intervention aimed at (challenging selfexempting beliefs about smoking and increasing risk perceptions in order to increase the desire to stop smoking) can only be achieved through intensive counselling and not through brief advice, which was initially hypothesised [17].
A major limitation of the present study is the small sample size. Approximately 30 fewer participants were included in the experimental group and control group 1 than expected according to the sample size calculation. Therefore, the results from the study are not conclusive. With regard to the comparison between the experimental group and control group 2, statistically significant differences were found in abstinence rates at week 5 and from week 5-26. However, the authors were unable to find a significant difference in abstinence rates from week 5-52. The study was not sufficiently powered to detect the observed difference of $5 \%$.

In conclusion, the present study did not provide evidence that confronting smokers who are interested in smoking cessation with previously undetected chronic obstructive pulmonary disease increases long-term smoking cessation rates. Confrontational counselling may have short-term effects, but these diminish during the first year after initial counselling treatment. The high failure rates dramatically emphasise the difficulty tobacco-addicted smokers experience with quitting smoking and highlight the need for treating tobacco addiction as a chronic relapsing disorder and to match it with an appropriate and tailored amount of care. This is especially indicated in smokers with respiratory disease, who have a more urgent need to stop smoking. Future research should investigate whether repeated counselling sessions during a longer follow-up period can consolidate an initial short-term effect and, therefore, increase long-term smoking cessation rates.

\section{ACKNOWLEDGEMENTS}

The authors gratefully acknowledge the contributions of: K. van der Meer (telephone screening and logistics; Dept of General Practice, School for Public Health and Primary Care (CAPHRI), Maastricht University Medical Centre, Maastricht, Germany); A. van de Voorde, E. de Goeij (spirometry; both Dept of General Practice, School for Public Health and Primary Care (CAPHRI)), M. Vaassen, H. Bastiaens, R. Romers, T. Sales and K. Frambach (respiratory nurses, all Dept of Respiratory Medicine, Maastricht University Medical Centre); M. Aussems (validation of spirometric test results; Dept of Respiratory Medicine); W. de Goeij (database construction; Infoficiency Ltd); G. Driessen and P. Rinkens (data cleaning; both Dept of General Practice, School for Public Health and Primary Care (CAPHRI)); L. Maas (cotinine analyses; Dept of Health Risk Analysis and Toxicology, Maastricht University Medical Centre); H. Boersma (pharmacist; Dept of Clinical Pharmacy and Toxicology, Maastricht University Medical Centre); and A. Kester (statistical advice; Dept of Methodology and Statistics, Maastricht University Medical Centre).

\section{REFERENCES}

1 Global Initiative for Chronic Obstructive Lung Disease. Global Strategy for the Diagnosis, Management and Prevention of Chronic Obstructive Pulmonary Disease. NHLBI/WHO Workshop Report. Reviewed edition. 2006. www.goldcopd.com/Guidelineitem.asp? $11=2 \& 12=1 \&$ intId =996 Date last accessed: November 25, 2008.

2 World Health Organization, The World Health Report 2002. Reducing Risks, Promoting Healthy Life. Geneva, World Health Organization, 2002. 
3 Murray CJ, Lopez AD. Alternative projections of mortality and disability by cause 1990-2020: Global Burden of Disease Study. Lancet 1997; 349: 1498-1504.

4 Pauwels RA, Buist AS, Calverley PM, Jenkins CR, Hurd SS. Global strategy for the diagnosis, management, and prevention of chronic obstructive pulmonary disease. NHLBI/WHO Global Initiative for Chronic Obstructive Lung Disease (GOLD) Workshop summary. Am J Respir Crit Care Med 2001; 163: 1256-1276.

5 Anthonisen NR, Connett JE, Kiley JP, et al. Effects of smoking intervention and the use of an inhaled anticholinergic bronchodilator on the rate of decline of FEV1. The Lung Health Study. J Am Med Assoc 1994; 272: 1497-1505.

6 Pauwels PRA, Rabe KF. Burden and clinical features of chronic obstructive pulmonary disease (COPD). Lancet 2004; 364: 613-620.

7 van Schayck CP, van der Heijden FMMA, van den Boom G, Tirimanna PRS, van Herwaarden CLA. Underdiagnosis of asthma: is the doctor or the patient to blame? The DIMCA project. Thorax 2000; 55: 562-565.

8 Boushey H, Enright P, Samet J. Spirometry for chronic obstructive pulmonary disease case finding in primary care? Am J Respir Crit Care Med 2005; 172: 1481-1482.

9 Enright PL, Studnicka M, Zielinski J. Spirometry to detect and manage chronic obstructive pulmonary disease and asthma in the primary care setting. In: Gosselink R, Stam H, eds. Lung Function Testing. Eur Respir Mon 2005; 31: 1-14.

10 White P. Should we use spirometry in the early detection of COPD? Eur Respir J 2005; 26: 558-559.

11 Wilt TJ, Niewoehner D, Kane RL, MacDonald R, Joseph AM. Spirometry as a motivational tool to improve smoking cessation rates: a systematic review of the literature. Nicotine Tob Res 2007; 9: 21-32.

12 Wilt TJ, Niewoehner D, Kim C-B, et al. Use of Spirometry for Case Finding, Diagnosis, and Management of Chronic Obstructive Pulmonary Disease (COPD). Evidence Report/ Technology Assessment No. 121 (Prepared by the Minnesota Evidence-based Practice Center under Contract No. 290-020009.) AHRQ Publication No. 05-E017-2. Rockville, Agency for Healthcare Research and Quality, 2005.

13 Enright P. Does screening for COPD by primary care physicians have the potential to cause more harm than good? Chest 2006; 129: 833-835.

14 McBride CM, Emmons KM, Lipkus IM. Understanding the potential of teachable moments: the case of smoking cessation. Health Educ Res 2003; 18: 156-170.

15 Aveyard P, Brown K, Saunders C, et al. Weekly versus basic smoking cessation support in primary care: a randomised controlled trial. Thorax 2007; 62: 898-903.

16 Parkes G, Greenhalgh T, Griffin M, Dent R. Effect on smoking quit rate of telling patients their lung age: the Step2quit randomised controlled trial. BMJ 2008; 336: 598-600.

17 Kotz D, Huibers MJ, Vos R, van Schayck CP, Wesseling G. Principles of confrontational counselling in smokers with chronic obstructive pulmonary disease (COPD). Med Hypotheses 2008; 70: 384-386.

18 Kotz D, Wesseling GJ, Huibers MJH, van Schayck CP. Efficacy of confrontational counselling for smoking cessation in smokers with previously undiagnosed mild to moderate airflow limitation: study protocol of a randomized controlled trial. BMC Public Health 2007; 7: 332.

19 Zelen M. A new design for randomized clinical trials. $N$ Engl J Med 1979; 300: 1242-1245.

20 Zelen M. Randomized consent designs for clinical trials: an update. Stat Med 1990; 9: 645-656.

21 Torgerson DJ, Roland M. What is Zelen's design? BMJ 1998; 316: 606.

22 Miller MR, Hankinson J, Brusasco V, et al. Standardisation of spirometry. Eur Respir J 2005; 26: 319-338.

23 Miller MR, Crapo R, Hankinson J, et al. General considerations for lung function testing. Eur Respir J 2005; 26: 153-161.

24 STIVORO. De L-MIS: stoppen met roken voor longpatiënten. Handleiding voor longverpleegkundigen. [The L-MIS: smoking cessation in lung patients. Strategy for respiratory nurses]. Den Haag, STIVORO, 2003.

25 Fletcher C, Peto R. The natural history of chronic airflow obstruction. Brit Med J 1977; 1: 1645-1648.

26 Hughes JR, Stead LF, Lancaster T. Antidepressants for smoking cessation. Cochrane Database of Syst Rev 2007; 1: CD000031.

27 Wagena EJ, Knipschild P, Zeegers MPA. Should nortriptyline be used as a first-line aid to help smokers quit? Results from a systematic review and meta-analysis. Addiction 2005; 100: 317-326.

28 Pieterse ME, Seydel ER, DeVries H, Mudde AN, Kok GJ, Halpern MT. Effectiveness of a minimal contact smoking cessation program for Dutch general practitioners: a randomized controlled trial. Prev Medicine 2001; 32: 182-190.

29 SRNT Subcommittee on Biochemical Verification, Biochemical verification of tobacco use and cessation. Nicotine Tob Res 2002; 4: 149-159.

30 Pocock SJ. Clinical Trials. A Practical Approach. Chichester, John Wiley \& Sons, 1983.

31 Heatherton TF, Kozlowski LT, Frecker RC, Fagerström KO. The Fagerström Test for Nicotine Dependence: a revision of the Fagerström Tolerance Questionnaire. $\mathrm{Br}$ J Addict 1991; 86: 1119-1127.

32 van der Molen T, Willemse BWM, Schokker S, ten Hacken NHT, Postma DS, Juniper EF. Development, validity and responsiveness of the Clinical COPD Questionnaire. Health Qual Life Outcomes 2003; 1: 13.

33 van der Molen T, Willemse BW, Schokker S, Postma DS, Juniper EF. Development and cross sectional validity of the COPD symptom control questionnaire. Prim Care Respir J 2001; 10: 75 .

34 Williams JEA, Singh SJ, Sewell L, Guyatt GH, Morgan MDL. Development of a self-reported Chronic Respiratory Questionnaire (CRQ-SR). Thorax 2001; 56: 954-959.

35 Guyatt GH, Berman LB, Townsend M, Pugsley SO, Chambers LW. A measure of quality of life for clinical trials in chronic lung disease. Thorax 1987; 42: 773-778.

36 Caponnetto P, Polosa R. Common predictors of smoking cessation in clinical practice. Respir Med 2008; 102: 1182-1192.

37 Zigmond AS, Snaith RP. The hospital anxiety and depression scale. Acta Psyciatr Scan 1983; 67: 361-370.

38 Bednarek M, Gorecka D, Wielgomas J, et al. Smokers with airway obstruction are more likely to quit smoking. Thorax 2006; 61: 869-873. 
39 Kotz D, van Schayck CP, Huibers MJ, et al. Assessing the efficacy of spirometry for smoking cessation. Thorax 2007; 62: 742.

40 Hughes J, Keely J, Niaura R, Ossip-Klein D, Richmond R, Swan G. Measures of abstinence in clinical trials: issues and recommendations. Nicotine Tob Res 2003; 5: 13-26.

41 Tashkin D, Kanner R, Bailey W. et al. Smoking cessation in patients with chronic obstructive pulmonary disease: a double-blind, placebo-controlled, randomised trial. Lancet 2001; 357: 1571-1575.

42 Wagena EJ, Knipschild PG, Huibers MJH, Wouters EFM, van Schayck CP. Efficacy of bupropion and nortriptyline for smoking cessation among people at risk for or with chronic obstructive pulmonary disease. Arch Intern Med 2005; 165: 2286-2292. 